\title{
AN APPLICATION OF MACAULAY'S ESTIMATE TO SUMS OF SQUARES PROBLEMS IN SEVERAL COMPLEX VARIABLES
}

\author{
DUSTY GRUNDMEIER AND JENNIFER HALFPAP KACMARCIK
}

(Communicated by Mei-Chi Shaw)

\begin{abstract}
Several questions in complex analysis lead naturally to the study of bihomogeneous polynomials $r(z, \bar{z})$ on $\mathbb{C}^{n} \times \mathbb{C}^{n}$ for which $r(z, \bar{z})\|z\|^{2 d}=$ $\|h(z)\|^{2}$ for some natural number $d$ and a holomorphic polynomial mapping $h=\left(h_{1}, \ldots, h_{K}\right)$ from $\mathbb{C}^{n}$ to $\mathbb{C}^{K}$. When $r$ has this property for some $d$, one seeks relationships between $d, K$, and the signature and rank of the coefficient matrix of $r$. In this paper, we reformulate this basic question as a question about the growth of the Hilbert function of a homogeneous ideal in $\mathbb{C}\left[z_{1}, \ldots, z_{n}\right]$ and apply a well-known result of Macaulay to estimate some natural quantities.
\end{abstract}

\section{INTRODUCTION}

This paper explores the use of commutative algebra in the study of Hermitian sums of squares problems in several complex variables. Suppose $r(z, \bar{z})$ is a bihomogeneous polynomial of bi-degree $(m, m)$ on $\mathbb{C}^{n} \times \mathbb{C}^{n}$. It is a well-known result of Quillen Qui68, proved independently by Catlin and D'Angelo CD97, that if $r$ is positive on the unit sphere $S^{2 n-1}$, then there exists a natural number $d$ such that $r(z, \bar{z})\|z\|^{2 d}$ can be written as a sum of squares; that is,

$$
r(z, \bar{z})\|z\|^{2 d}=\sum_{k=1}^{K}\left|h_{k}(z)\right|^{2}=\|h(z)\|^{2}
$$

for holomorphic polynomials $h_{k}: \mathbb{C}^{n} \rightarrow \mathbb{C}$. See CD99 for an interpretation of this result in terms of holomorphic line bundles and see [D'A11 for an interpretation as a Hermitian analogue of Hilbert's 17th problem. Recent investigations ([DZ13], [TY06]) have sought results linking the minimum $d$ for which $r(z, \bar{z})\|z\|^{2 d}$ is a squared norm to properties of $r$. This minimum $d$ must depend on the size of $r$ on the sphere and not just on the dimension $n$ and the bi-degree $(m, m)$, as the example

$$
\left(\left|z_{1}\right|^{2}-\left|z_{2}\right|^{2}\right)^{2}+\varepsilon\left|z_{1} z_{2}\right|^{2}
$$

shows; in this example, the minimum $d$ tends to infinity as $\varepsilon$ tends to 0 .

Our approach is algebraic. Given a bihomogeneous $r$ with signature $(P, N)$ positive on the sphere, there is a $d$ for which (11) holds. We seek to understand the relationship among the dimension $n$, the bi-degree $(m, m)$, the natural number $d$, and the signature $(P, N)$. The inequalities we obtain can be viewed in several ways;

Received by the editors March 31, 2013 and, in revised form, August 2, 2013.

2010 Mathematics Subject Classification. Primary 13D40, 32A17, 32H99.

The first author was partially supported by NSF RTG grant DMS-1045119.

The second author was supported in part by NSF grant DMS 1200815. 
they say, for a fixed $r$ and $d$ for which (1) holds, what inequality the signature $(P, N)$ must satisfy. Alternatively, the inequalities give, for a bihomogeneous $r$ positive on the sphere with signature $(P, N)$, a lower bound on $D_{d}$, and hence on the $d$ for which (11) holds.

We reformulate our basic question as a question about the growth of the Hilbert function of a homogeneous ideal in the polynomial ring $\mathbb{C}\left[z_{1}, \ldots, z_{n}\right]$, and we use a well-known estimate of Macaulay (Theorem 1 below). Ours is not the first paper to apply such results from commutative algebra to questions in several complex variables; Grundmeier, Lebl, and Vivas use a similar set of ideas in GLV11] to prove a rigidity theorem for CR mappings of hyperquadrics. Our goal is not primarily to obtain specific inequalities but rather to illustrate how a set of ideas from this area of algebra can be brought to bear on questions arising in several complex variables. We end the paper with a section exploring more fully these connections with several complex variables and CR geometry.

\section{Definitions AND A KeY LEMma}

Let $r$ be a real-valued bihomogeneous polynomial of bi-degree $(m, m)$ on $\mathbb{C}^{n} \times \mathbb{C}^{n}$; thus

$$
r(z, \bar{z})=\sum_{|\alpha|=|\beta|=m} c_{\alpha \beta} z^{\alpha} \bar{z}^{\beta}
$$

for some Hermitian matrix $\left(c_{\alpha \beta}\right)$. Each such polynomial has a holomorphic decomposition

$$
r(z, \bar{z})=\|f(z)\|^{2}-\|g(z)\|^{2}=\sum_{j=1}^{P}\left|f_{j}(z)\right|^{2}-\sum_{k=1}^{N}\left|g_{k}(z)\right|^{2}
$$

where the $f_{j}, g_{k}$, are holomorphic homogeneous polynomials of degree $m$ and the set $\left\{f_{j}, g_{k}: 1 \leq j \leq P, 1 \leq k \leq N\right\}$ is linearly independent. This decomposition is obtained by diagonalizing the coefficient matrix $\left(c_{\alpha \beta}\right)$. Thus, although the polynomials appearing in the holomorphic decomposition of a given $r$ are not uniquely determined, the signature pair $(P, N)$ and the rank $R=P+N$ are. Observe that $r$ has signature pair $(P, 0)$ if and only if it is itself a squared norm. The idea of studying real polynomials on $\mathbb{C}^{n}$ through Hermitian linear algebra has been used extensively by D'Angelo; see Chapter VI in D'A02] and D'A93.

Our results concern the dimensions of various vector spaces naturally associated with $r$. Let $\mathcal{P}_{k}$ be the space of homogeneous polynomials of degree $k$ in $z_{1}, \ldots, z_{n}$. Let $D_{k}=\left(\begin{array}{c}k+n-1 \\ k\end{array}\right)$, which is the dimension of this space. For any mapping $p=$ $\left(p_{1}, \ldots, p_{L}\right): \mathbb{C}^{n} \rightarrow \mathbb{C}^{L}$ with components in $\mathcal{P}_{k}$, let $V_{p}$ be the subspace of $\mathcal{P}_{k}$ spanned by the components of $p$. If $q: \mathbb{C}^{n} \rightarrow \mathbb{C}^{L^{\prime}}$ is a second mapping, then the mappings $p \oplus q$ and $p \otimes q$ satisfy $\|p \oplus q\|^{2}=\|p\|^{2}+\|q\|^{2}$ and $\|p \otimes q\|^{2}=\|p\|^{2}\|q\|^{2}$. We can think of $z$ as the mapping whose components are the coordinate functions $z_{1}, \ldots, z_{n}$; Thus $z^{\otimes d}$ is the tensor product of this mapping with itself $d$ times.

The following lemma, which appears in a more general form in D'A05, is used heavily.

Lemma 1. Let $r$ be bihomogeneous of bi-degree $(m, m)$ with $r(z, \bar{z})\|z\|^{2 d}=\|h(z)\|^{2}$. Let $F=f \otimes z^{\otimes d}$ and $G=g \otimes z^{\otimes d}$. Then $V_{G} \subseteq V_{F}$. 
Proof. Note that

$$
r(z, \bar{z})\|z\|^{2 d}=\left(\|f\|^{2}-\|g\|^{2}\right)\|z\|^{2 d}=\left\|f \otimes z^{\otimes d}\right\|^{2}-\left\|g \otimes z^{\otimes d}\right\|^{2} .
$$

Because $r(z, \bar{z})\|z\|^{2 d}=\|h(z)\|^{2}$,

$$
\left\|f \otimes z^{\otimes d}\right\|^{2}=\left\|g \otimes z^{\otimes d}\right\|^{2}+\|h\|^{2}=\left\|\left(g \otimes z^{\otimes d}\right) \oplus h\right\|^{2},
$$

that is, $\|F(z)\|^{2}=\|(G \oplus h)(z)\|^{2}$. Let $L$ be a natural number such that the rank of $F$ and the rank of $G \oplus h$ are at most $L$. By adding identically zero components as needed, we obtain two mappings $F \oplus 0$ and $G \oplus h \oplus 0$ from $\mathbb{C}^{n}$ to $\mathbb{C}^{L}$ with the same squared norm. Thus there exists a unitary transformation $U$ on $\mathbb{C}^{L}$ such that $U(F \oplus 0)=(G \oplus h \oplus 0)$. It follows that the components of the mapping $G$ are in the linear span of the components of the mapping $F$.

To this point, for a mapping $h$ whose components are homogeneous of degree $m$, we have simply considered the vector subspace $V_{h}$ of $\mathcal{P}_{m}$ spanned by the components of $h$. We change our perspective somewhat; we think of $S=\mathbb{C}\left[z_{1}, \ldots, z_{n}\right]$ as a graded ring, graded by degree. We let $I_{h}$ as the homogeneous ideal generated by the components of $h . I_{h}$ is a graded $S$-module, with its degree $k$ component denoted by $\left(I_{h}\right)_{k}$. In particular, its first non-trivial component, $\left(I_{h}\right)_{m}$, is the vector space $V_{h}$ discussed above. The Hilbert function of $I_{h}$ is

$$
H_{I_{h}}(k)=\operatorname{dim}_{\mathbb{C}}\left(I_{h}\right)_{k} .
$$

We will make use of Macaulay's estimate on the growth of a homogeneous ideal. Macaulay's result gives an upper bound for the Hilbert function for $S / I_{h}$ and hence a lower bound for the Hilbert function for $I_{h}$.

Definition 1. Let $c$ and $\nu$ be positive integers. The $\nu$-th Macaulay representation of $c$ is the (unique) way of writing

$$
c=\left(\begin{array}{c}
k_{\nu} \\
\nu
\end{array}\right)+\left(\begin{array}{c}
k_{\nu-1} \\
\nu-1
\end{array}\right)+\ldots+\left(\begin{array}{c}
k_{J} \\
J
\end{array}\right)
$$

where $k_{\nu}>k_{\nu-1}>\ldots>k_{J} \geq J>0$. We also write

$$
c^{<\nu>}=\left(\begin{array}{c}
k_{\nu}+1 \\
\nu+1
\end{array}\right)+\left(\begin{array}{c}
k_{\nu-1}+1 \\
\nu
\end{array}\right)+\ldots+\left(\begin{array}{c}
k_{J}+1 \\
J+1
\end{array}\right) .
$$

See Gre10] and Gre89] for a more extensive discussion of these ideas, including proofs of the uniqueness of the $\nu$-th Macaulay representation of a positive integer and of the elementary properties of the function $c \mapsto c^{<\nu>}$. We need only two such properties:

Lemma 2. Let $b, c$, and $\nu$ be positive integers.

(1) If $b<c$, then $b^{<\nu>}<c^{<\nu>}$.

(2) For any $k \in \mathbb{N}$ with $k<c,(c-k)^{<\nu>} \leq c^{<\nu>}-k$.

Theorem 1 (Macaulay's estimate on the growth of an ideal Mac27]). Let I be an ideal in $S=\mathbb{C}\left[z_{1}, \ldots, z_{n}\right]$ whose generators are homogeneous polynomials (not necessarily all of the same degree). Then

$$
H_{S / I}(k+1) \leq H_{S / I}(k)^{<k>} .
$$


Elementary linear algebra shows that

$$
H_{S / I}(k)+H_{I}(k)=\left(\begin{array}{c}
k+n-1 \\
k
\end{array}\right)=D_{k} .
$$

\section{The MAIN THEOREM AND ITS COROLlaRies}

In this section, we establish our main result concerning signatures of real polynomials on $\mathbb{C}^{n}$. See HL13 for a discussion of connections of this problem to CR geometry and for constructions of families of polynomials illustrating the extent to which some such inequalities are sharp. Additional comments on the sharpness of these inequalities appear at the end of the section.

Theorem 2. Let $r$ be bihomogeneous of bi-degree $(m, m)$ with signature $(P, N)$ and rank $R$. Suppose $r(z, \bar{z})\|z\|^{2 d}$ is a squared norm. Then

$$
P \geq \frac{D_{m+d}-\left[\left[H(m)^{<m>}\right]^{<m+1>\ldots}\right]^{<m+d-1>}}{D_{d}}
$$

for $H(m)=D_{m}-R$. If the m-th Macaulay representation of $D_{m}-R$ is $\sum_{j=J}^{m}\left(\begin{array}{c}k_{j} \\ j\end{array}\right)$, this is equivalent to

$$
P \geq \frac{D_{m+d}-\sum_{j=J}^{m}\left(\begin{array}{c}
k_{j}+d \\
j+d
\end{array}\right)}{D_{d}} .
$$

Proof. We obtain upper and lower bounds for $H_{I_{f}}(m+d)$, which equals $H_{I_{f \oplus g}}(m+d)$ since, by Lemma 1, the components of $I_{f}$ and $I_{f \oplus g}$ in degree $m+d$ are the same. Since $V_{f}=\left(I_{f}\right)_{m}$ has as a basis the $P$ generators $f_{j}$ of the ideal $I_{f}$, $\left(I_{f}\right)_{m+d}$ is spanned by the polynomials $z^{\alpha} f_{j}$ for $|\alpha|=d$. We thus have the upper bound

$$
H_{I_{f}}(m+d) \leq D_{d} P .
$$

We use Theorem 1 to obtain an upper bound on $H_{S / I_{f \oplus g}}(m+d)$. To simplify notation, we drop the subscript on $H$ when discussing the Hilbert function for $S / I_{f \oplus g}$. Since $H_{I_{f \oplus g}}(m)=R$,

$$
H(m)=D_{m}-R
$$

Suppose the $m$-th Macaulay representation of $D_{m}-R$ is $\sum_{j=J}^{m}\left(\begin{array}{c}k_{j} \\ j\end{array}\right)$. According to Macaulay's estimate,

$$
H(m+1) \leq H(m)^{<m>}=\sum_{j=J}^{m}\left(\begin{array}{c}
k_{j}+1 \\
j+1
\end{array}\right) .
$$

Applying the result again and using Lemma 2 gives

$$
H(m+2) \leq H(m+1)^{<m+1>} \leq\left[H(m)^{<m>}\right]^{<m+1>}=\left(\sum_{j=J}^{m}\left(\begin{array}{c}
k_{j}+1 \\
j+1
\end{array}\right)\right)^{<m+1>}
$$

Since the expression in parentheses is already in the form of the $(m+1)$ st Macaulay representation of an integer, the full expression on the right can be easily evaluated and we obtain:

$$
H(m+2) \leq \sum_{j=J}^{m}\left(\begin{array}{c}
k_{j}+2 \\
j+2
\end{array}\right)
$$


Iterating gives

$$
H(m+d) \leq\left[\left[H(m)^{<m>}\right]^{<m+1>} \ldots\right]^{<m+d-1>}=\sum_{j=J}^{m}\left(\begin{array}{c}
k_{j}+d \\
j+d
\end{array}\right)
$$

Since $H_{I_{f \oplus g}}(m+d)=D_{m+d}-H(m+d)$, the result follows.

We give two corollaries. In both cases, a simple direct proof also exists that does not require Macaulay's estimate. We sketch both arguments for Corollary 2 , Furthermore, since we always use the upper bound

$$
H_{I_{f}}(m+d) \leq D_{d} P
$$

when $f$ has $P$ components, we need only describe how we obtain the lower bounds.

Corollary 1. Let $r$ be bihomogeneous of bi-degree $(m, m)$ with signature pair $(P, N)$ and rank $R$. If $r(z, \bar{z})\|z\|^{2 d}$ is a squared norm, then

$$
N \leq(P-1)\left(D_{d}-1\right) .
$$

Proof. First, observe that

$$
D_{m}=\sum_{k=0}^{m}\left(\begin{array}{c}
k+n-2 \\
k
\end{array}\right) .
$$

This follows by counting the monomials of degree $m$ in $n$ variables in two ways. On the one hand, this number is $D_{m}$. On the other hand, we may count the monomials of degree $m$ involving $x_{1}^{m-k}$ for $0 \leq k \leq m$, which gives $\left(\begin{array}{c}k+n-2 \\ k\end{array}\right)$. Sum over $k$.

By Theorem 2.

$$
D_{d} P \geq D_{m+d}-\left[\left[\left[D_{m}-R\right]^{<m>}\right]^{<m+1>\ldots}\right]^{<m+d-1>} .
$$

By statement (2) in Lemma 2 and the above expression for $D_{m}$,

$$
\begin{aligned}
& D_{m+d}-\left[\left[\left[D_{m}-R\right]^{<m>}\right]^{<m+1>} \ldots\right]^{<m+d-1>} \\
& \quad \geq D_{m+d}-\left[\left[\left[D_{m}-1\right]^{<m>}\right]^{<m+1>\ldots]^{<m+d-1>}+R-1}+[]^{<}\right] \\
& \quad=\sum_{k=0}^{m+d}\left(\begin{array}{c}
k+n-2 \\
k
\end{array}\right)-\left[\left[\sum_{k=1}^{m}\left(\begin{array}{c}
k+n-2 \\
k
\end{array}\right)\right]^{<m>}\right]^{<m+1>}+R-1 \\
& =\sum_{k=0}^{m+d}\left(\begin{array}{c}
k+n-2 \\
k
\end{array}\right)-\sum_{k=1}^{m}\left(\begin{array}{c}
k+n-2+d \\
k+d
\end{array}\right)+R-1 \\
& =\sum_{k=0}^{d}\left(\begin{array}{c}
k+n-2 \\
k
\end{array}\right)+R-1 \\
& =D_{d}+R-1 .
\end{aligned}
$$

Thus

which is equivalent to

$$
\frac{P}{R} \geq \frac{D_{d}+R-1}{D_{d} R}
$$

$$
N \leq(P-1)\left(D_{d}-1\right)
$$


Remark 1. The inequality in Corollary 1 immediately implies $N \leq P\left(D_{d}-1\right)$. The latter appears in Theorem 1.1(i) in [HL13. There, the proof given uses Hermitian linear algebra; although that proof is elementary, it is not straight-forward. On the other hand, the above proof uses a simple argument to prove a much stronger result, and hence illustrates the value of bringing techniques from commutative algebra to bear on such problems.

Corollary 2. Let $r$ be bihomogeneous of bi-degree $(m, m)$ with $r(z, \bar{z})\|z\|^{2 d}$ a squared norm and $R=D_{m}$ (which is the largest rank possible for this $m$ and $n)$. Then

$$
P \geq \frac{D_{m+d}}{D_{d}}
$$

Remark 2. Suppose $n=2$. Then (18) gives $P / R \geq(m+d+1) /(d+1)(m+1)$. See HL13 for an example of a polynomial in two variables for which this ratio is achieved.

Proof. To obtain a proof using Theorem 2, we need only observe that $D_{m}-R=0$ and so the numerator of (9) reduces to $D_{m+d}$.

Next we give a direct proof for the lower bound on $H_{I_{f}}(m+d)=H_{I_{f \oplus g}}(m+d)$. If the rank of $r$ is $D_{m}=\operatorname{dim} \mathcal{P}_{m}$, then in fact the components of $f \oplus g$ span $\mathcal{P}_{m}$. Thus the components of $F \oplus G$ span $\mathcal{P}_{m+d}$, i.e.,

$$
H_{I_{f \oplus g}}(m+d)=D_{m+d} .
$$

Then

$$
P \geq \frac{D_{m+d}}{D_{d}}
$$

We examine our method of proof more closely: We obtain the upper bound $H_{I_{f}}(m+d) \leq D_{d} P$ because the $D_{d} P$ functions $z^{\alpha} f_{j}$ for $1 \leq j \leq P$ and $|\alpha|=d$ span $\left(I_{f}\right)_{m+d}$. We thus have equality if and only if these functions are linearly independent. On the other hand, we obtain our lower bound for $H_{I_{f \oplus g}}(m+d)$ using Macaulay's theorem. It is considerably more difficult to say when equality holds here. (See Green Gre10 for a more detailed discussion.) We make a definition:

Definition 2. Let $I$ be a monomial ideal. $I$ is a lex segment ideal in degree $s$ if $I_{s}$ is spanned by the first $\operatorname{dim}\left(I_{s}\right)$ monomials of degree $s$ in lexicographic order.

The following results are known.

Proposition 1 (Macaulay). If $I$ is a lex segment ideal in degree $s$ and has no generators in degree $s+1, H_{S / I}(s+1)=H_{S / I}(s)^{<s>}$.

Theorem 3 (Gotzmann's Persistence Theorem). Let I be a homogeneous ideal generated in degree $\leq s$. If $H_{S / I}(s+1)=H_{S / I}(s)^{<s>}$, then $H_{S / I}(k+1)=H_{S / I}(k)^{<k>}$ for all $k \geq s$.

When the $R$ generators of a monomial ideal $I$ are all of the same degree $m$, as is the case with all of our ideals, $I$ is a lex segment ideal in all degrees if and only if the generators are the first $R$ monomials of degree $m$ in lexicographic order. Thus we have already encountered a simple lex segment ideal in Corollary 2.

With lexicographic order on the monomials and for a homogeneous ideal $I$, let $\operatorname{in}(I)$ be its initial ideal. This is a monomial ideal. Then $I$ and $\operatorname{in}(I)$ have the same 
Hilbert function in all degrees. We immediately obtain the following proposition describing a hypothesis under which we obtain an equality upon application of Macaulay's theorem.

Proposition 2. Let $r=\|f\|^{2}-\|g\|^{2}$ be as in Theorem 2, Suppose $\operatorname{in}\left(I_{f \oplus g}\right)$ is a lex segment ideal. Then

$$
H_{I_{f \oplus g}}(m+d)=D_{m+d}-\left[\left[H(m)^{<m>}\right]^{<m+1>} \ldots\right]^{<m+d-1>} .
$$

We now consider whether Corollaries 1 and 2 are sharp for all $n, d$, and $m$. In Corollary $2, I_{f \oplus g}$ is a lex segment ideal and $H_{I_{f \oplus g}}(m+d)=D_{m+d}$, i.e., we have an equality rather than merely a lower bound. Thus we ask whether we can have equality in the upper bound $H_{I_{f \oplus g}}(m+d) \leq D_{d} P$. In the monomial case the question is whether there are $D_{m+d} / D_{d}$ monomials $x^{\beta}$ such that the $D_{m+d}$ monomials $x^{\alpha+\beta}$ for $|\alpha|=d$ are distinct. When $n=2$, such a collection of monomials sometimes exists. Indeed, take $d$ a natural number and consider $m=k(d+1)$ for some natural number $k$. Then the $k+1$ monomials $x^{m-j(d+1)} y^{j(d+1)}, 0 \leq j \leq k$ have this property.

On the other hand, for $n>2$, even when $d=1$, it is not possible to find a collection of monomials $A=\left\{x^{\beta}: \beta \in \mathcal{B}\right\}$ such that the $3|A|$ monomials $x^{\beta+\mathbf{e}^{j}}$ for $\beta \in \mathcal{B}$ and $1 \leq j \leq 3\}$ are independent and span the space of homogeneous polynomials of degree $m+1$. Indeed, let $m \geq 2$ be arbitrary (the case $m=1$ is trivial). Since $x_{1}^{m+1}$ must be one of the $x^{\beta+\mathbf{e}^{j}}$, necessarily $x_{1}^{m} \in A$. Next, one of $x_{1}^{m-1} x_{2}$ and $x_{1}^{m-2} x_{2}^{2}$ is in $A$. If it were the former, $x_{1}^{m} x_{2}$ would arise from two different monomials in $A$. Thus we suppose it is $x_{1}^{m-2} x_{2}^{2}$. Similarly, suppose $x_{1}^{m-2} x_{3}^{2}$. None of these three monomials gives rise to $x_{1}^{m-1} x_{2} x_{3}$. Thus $A$ must contain one of $x_{1}^{m-1} x_{2}, x_{1}^{m-1} x_{3}$, or $x_{1}^{m-2} x_{2}, x_{3}$. The first two possibilities have already been eliminated. But if $x_{1}^{m-2} x_{2} x_{3}$ is in $A, x_{1}^{m-2} x_{2}^{2} x_{3}$ comes from both $x_{1}^{m-2} x_{2} x_{3}$ and $x_{1}^{m-2} x_{2}^{2}$ in $A$.

To summarize the above discussion, we see that even in the case in which the components of $f$ and $g$ are monomials, if $n>2$ the inequality in Corollary 2 is not sharp.

Next we consider Corollary 1, which implies

$$
\frac{N}{P} \leq D_{d}-1
$$

In [HL13, it is shown that this inequality is essentially sharp for $d=1$ for all $n$. This is not inconsistent with the above discussion; the family of polynomials constructed there has several interesting properties.

(1) $I_{f \oplus g}$ is a monomial ideal and includes all monomials of degree $m$ in $n$ variables. Thus it is a lex segment ideal and $H_{I_{f \oplus g}}(m+d)=D_{m+d}$.

(2) Consider the set $B$ of all components $x^{\beta}$ of $f$ for which $\beta_{j} \geq 1$ for all $1 \leq j \leq n$. The monomials $x^{\beta+\mathbf{e}^{k}}$ for $x^{\beta} \in A$ and $1 \leq k \leq n$ are all distinct.

Thus although there are components of $f$ giving rise to the same monomial of degree $m+1$, the proportion of such components of $f$ goes to zero as $m$ increases.

\section{Polynomials With Small Rank}

Theorem 2 and its corollaries give lower bounds on $P / R$, and hence upper bounds on $N / P$. In a sense, these results say which signatures are possible if $r(z, \bar{z})\|z\|^{2 d}$ is 
a squared norm and $r$ has large rank; in HL13, those polynomials for which $P / R$ is close to $1 / D_{d}$ have large degree and rank. By contrast, our next two propositions explore the situation in which $r(z, \bar{z})\|z\|^{2 d}$ is a squared norm but the rank of $r$ and the ratio $N / P$ are small.

Proposition 3. If $r$ is the squared norm of a holomorphic homogeneous polynomial mapping, then either the rank $K$ of $r(z, \bar{z})\|z\|^{2 d}$ satisfies

$$
n P-\frac{P(P-1)}{2} \leq K \leq n P
$$

for some $P \in \mathbb{N}$ with $P<n$, or $K \geq n(n+1) / 2$.

Remark 3. In other words, for a fixed dimension $n$, if $r$ is a squared norm, not all ranks of $r(z, \bar{z})\|z\|^{2 d}$ are possible; the smallest possible (non-zero) rank is $n$, and the next smallest are $2 n-1$ and $2 n$. For example, when $n=3$, ranks 1,2 , and 4 are not possible.

Proof. If $r$ is a squared norm, so is $\tilde{r}(z, \bar{z})=r(z, \bar{z})\|z\|^{2(d-1)}$. Thus by replacing $r$ by $\tilde{r}$ if necessary, it suffices to prove the result for $d=1$.

Suppose $r$ is the squared norm of the homogeneous holomorphic mapping $f=$ $\left(f_{1}, \ldots, f_{P}\right)$ so that

$$
H_{I_{f}}(m+1) \leq n P .
$$

We use Macaulay's estimate to obtain a lower bound.

Suppose first that $P<n$. Then

$$
\begin{aligned}
\left(D_{m}-P\right)^{<m>} & =\left(\sum_{k=1}^{m}\left(\begin{array}{c}
k+n-2 \\
k
\end{array}\right)+1-P\right)^{<m>} \\
& =\left(\sum_{k=2}^{m}\left(\begin{array}{c}
k+n-2 \\
k
\end{array}\right)+\left(\begin{array}{c}
n-P \\
1
\end{array}\right)\right) \\
& =\sum_{k=2}^{m}\left(\begin{array}{c}
k+n-1 \\
k+1
\end{array}\right)+\left(\begin{array}{c}
n-P+1 \\
2
\end{array}\right) \\
& =\sum_{k=1}^{m+1}\left(\begin{array}{c}
k+n-2 \\
k
\end{array}\right)+\left(\begin{array}{c}
n-P+1 \\
2
\end{array}\right)-(n-1)-\left(\begin{array}{c}
n \\
2
\end{array}\right) \\
& =D_{m+1}-\left(\frac{n^{2}+n}{2}-\frac{(n-P+1)(n-P)}{2}\right) .
\end{aligned}
$$

We obtain the lower bound

$$
H_{I_{f}}(m+1) \geq \frac{n^{2}+n}{2}-\frac{(n-P+1)(n-P)}{2}=n P-\frac{P(P-1)}{2} .
$$

Thus for $P<n$,

$$
n P-\frac{P(P-1)}{2} \leq K \leq n P .
$$

Next suppose $P \geq n$. Then

$$
H_{S / I_{f}}(m+1) \leq\left(H_{S / I_{f}}(m)\right)^{<m>} \leq\left(D_{m}-n\right)^{<m>}=D_{m+1}-\frac{n(n+1)}{2},
$$

and $H_{I_{f}}(m+1) \geq n(n+1) / 2$. 
Finally, we consider $r$ of signature $(P, 1)$. In [HL13, the authors prove that if, in the holomorphic decomposition of such an $r$, the components of $f$ and $g$ are monomials, then $P \geq n$. Furthermore, it is clear that signature pair $(n, 1)$ is possible.

We consider this question in the general situation in which the components of $f$ and $g$ are arbitrary homogeneous polynomials of degree $m$. We use Macaulay's estimate to show that certain values for $\left(I_{f}\right)_{k}$ and $\left(I_{f \oplus g}\right)_{k}$ are not possible if these spaces are known to agree for $k \geq m+1$.

Proposition 4. Let $r$ be bihomogeneous of bi-degree $(m, m)$ with $r(z, \bar{z})\|z\|^{2} a$ squared norm. If $N=1$, then

$$
P^{2}+P \geq 2 n \text {. }
$$

Proof. Since $H_{I_{f}}(m)=P$ and $H_{I_{f \oplus g}}(m)=P+1, H_{S / I_{f \oplus g}}(m)=D_{m}-(P+1)$.

We know that it is possible to take $P=n$. Thus our concern in this proposition is whether the Macaulay estimates themselves rule out the possibility of smaller $P$. Thus we consider $P<n$ and compare upper and lower bounds for $H_{S / I_{f \oplus g}}(m+1)$. We expect to see that if $P$ is too small, the lower bound given by the Macaulay estimates will be larger than our known upper bound.

To begin, we need the $m$-th Macaulay representation of $D_{m}-(P+1)$, where $P<n$. We begin as in the previous proposition, writing

$$
\begin{aligned}
\left(D_{m}-(P+1)\right)^{<m>} & =\left(\sum_{k=1}^{m}\left(\begin{array}{c}
k+n-2 \\
k
\end{array}\right)-P\right)^{<m>} \\
& =\left(\sum_{k=2}^{m}\left(\begin{array}{c}
k+n-2 \\
k
\end{array}\right)+\left(\begin{array}{c}
n-1-P \\
1
\end{array}\right)\right)^{<m>}
\end{aligned}
$$

We consider two cases. First, suppose $P=n-1$. Then

$$
\begin{aligned}
\text { (25) } & =\sum_{k=2}^{m}\left(\begin{array}{c}
k+n-1 \\
k+1
\end{array}\right) \\
& =\sum_{k=1}^{m+1}\left(\begin{array}{c}
k+n-2 \\
k
\end{array}\right)-\left(\begin{array}{l}
n \\
2
\end{array}\right)-\left(\begin{array}{c}
n-1 \\
1
\end{array}\right) \\
& =D_{m+1}-\frac{n(n+1)}{2} \\
& =D_{m+1}-\frac{(P+1)(P+2)}{2} .
\end{aligned}
$$

For the second case, suppose $P<n-1$. We obtain

$$
\begin{aligned}
\text { (25) } & =\sum_{k=2}^{m}\left(\begin{array}{c}
k+n-1 \\
k+1
\end{array}\right)+\left(\begin{array}{c}
n-P \\
2
\end{array}\right) \\
& =D_{m+1}-\left(n+n P-\frac{P^{2}+P}{2}\right)
\end{aligned}
$$

Since for $P=n-1$

$$
n+n P-\frac{P^{2}+P}{2}=\frac{(P+1)(P+2)}{2},
$$


for any $P<n$ we obtain the lower bound

$$
H_{S / I_{f \oplus g}}(m+1)=n+n P-\frac{P^{2}+P}{2} .
$$

As always, we trivially have the upper bound

$$
H_{I_{f \oplus g}}(m+1) \leq n P .
$$

Thus necessarily

$$
n+n P-\frac{P(P+1)}{2} \leq n P .
$$

Thus we require

$$
2 n \leq P^{2}+P
$$

The two propositions of this section illustrate what information about bihomogeneous polynomials can be gained through the use of this algebraic tool; rather than establishing the existence of bihomogeneous polynomials with certain algebraic properties, Macaulay's estimate allows us to show that certain scenarios are impossible because of necessary relationships between the dimensions of various vector spaces naturally associated with a polynomial.

\section{Connections With mapping PRoblems in CR Geometry}

Hermitian sums of squares arise in many places in several complex variables. In this section, we describe connections with CR geometry.

Given two real hypersurfaces in $\mathbb{C}^{n}$, a fundamental problem is to find and classify all CR mappings between them. A natural starting point is to study CR mappings between spheres. Thus if $h: S^{2 n-1} \rightarrow S^{2 N-1}$ is a CR mapping,

$$
\|h(z)\|^{2}-1=S(z, \bar{z})\left(\|z\|^{2}-1\right)
$$

and hence for CR mappings of spheres, Hermitian sums of squares naturally enter the problem. Huang Hua99 showed that $\operatorname{rank}\left(S(z, \bar{z})\|z\|^{2}\right) \geq n$. This result, known as "Huang's Lemma," is then used in many places in CR geometry (see for example [EHZ05], HJY13, and Ebe13] and the references therein). In particular, Huang Hua99] used this lemma to prove that if $f: S^{2 n-1} \rightarrow S^{2 N-1}$ is a $\mathcal{C}^{2}$-smooth CR mapping and $N<2 n-1, n>1$, then $f$ is equivalent to the linear map $z \mapsto(z, 0)$.

A natural generalization is to allow negative eigenvalues in the defining equations; namely, we consider the hyperquadrics given by

$$
Q(P, N)=\left\{z \in \mathbb{C}^{P+N}: \sum_{j=1}^{P}\left|z_{j}\right|^{2}-\sum_{j=P+1}^{P+N}\left|z_{j}\right|^{2}=1\right\} .
$$

Again many results are known in this situation. In particular, the first author Gru11 studied the possible target hyperquadrics of CR mappings from a sphere invariant under a group action. In this setting, one again naturally studies Hermitian sums of squares; however, we keep track of the signature pair instead of just the rank. That is, one studies the signature pairs of $S(z, \bar{z})\left(\|z\|^{2}-1\right)$ for particular $S(z, \bar{z})$.

The work of Catlin and D'Angelo CD96 gives another connection between CR geometry and the sorts of sums of squares problems considered here. Let $B_{n}$ denote 
the unit ball in $\mathbb{C}^{n}$, and suppose $f=\frac{p}{q}: \mathbb{C}^{n} \rightarrow \mathbb{C}^{N}$ is a rational mapping such that $f\left(\overline{B_{n}}\right) \subseteq B_{N}$. Catlin and D'Angelo show that it is a consequence of (11) that one can find a natural number $K$ and a polynomial mapping $g: \mathbb{C}^{n} \rightarrow \mathbb{C}^{K}$ such that $\frac{p \oplus g}{q}$ is a CR mapping from $S^{2 n-1}$ to $S^{2(N+K)-1}$. Thus the result of Quillen-CatlinD'Angelo gives a way to arbitrarily assign many of the components of CR mappings between spheres. In order to understand the relationships among the degree, the domain dimension, and the target dimension for CR mappings constructed in this manner, it is essential in (1) to understand the relationships among the bi-degree and signature of $r$, the natural number $d$, the dimension $n$, and the rank $K$ of the Hermitian squared norm. Some of these quantities certainly depend on analytic properties of $r$, as we pointed out in the introduction. Our purpose here has been to understand which relationships are purely algebraic.

\section{ACKNOWLEDGMENT}

We would like to thank John D'Angelo for encouraging us to pursue an algebraic reformulation of such questions and for many helpful conversations. We would also like to thank the referee for a number of suggestions that have significantly improved the manuscript.

\section{REFERENCES}

[CD96] David W. Catlin and John P. D'Angelo, A stabilization theorem for Hermitian forms and applications to holomorphic mappings, Math. Res. Lett. 3 (1996), no. 2, 149-166, DOI 10.4310/MRL.1996.v3.n2.a2. MR1386836 (97f:32025)

[CD97] David W. Catlin and John P. D'Angelo, Positivity conditions for bihomogeneous polynomials, Math. Res. Lett. 4 (1997), no. 4, 555-567, DOI 10.4310/MRL.1997.v4.n4.a11. MR $1470426(98 \mathrm{e}: 32023)$

[CD99] David W. Catlin and John P. D'Angelo, An isometric imbedding theorem for holomorphic bundles, Math. Res. Lett. 6 (1999), no. 1, 43-60, DOI 10.4310/MRL.1999.v6.n1.a4. MR 1682713 (2000g:32023)

[D'A93] John P. D'Angelo, Several complex variables and the geometry of real hypersurfaces, Studies in Advanced Mathematics, CRC Press, Boca Raton, FL, 1993. MR 1224231 (94i:32022)

[D'A02] John P. D'Angelo. Inequlaities from complex analysis. Carus Mathematical Monographs. MAA, 2002.

[D'A05] John P. D'Angelo, Complex variables analogues of Hilbert's seventeenth problem, Internat. J. Math. 16 (2005), no. 6, 609-627, DOI 10.1142/S0129167X05002990. MR2153486 (2006h:32004)

[D'A11] John P. D'Angelo, Hermitian analogues of Hilbert's 17-th problem, Adv. Math. 226 (2011), no. 5, 4607-4637, DOI 10.1016/j.aim.2010.12.013. MR2770459 (2012a:12001)

[DZ13] Alexis Drouot and Maciej Zworski, A quantitative version of Catlin-D'Angelo-Quillen theorem, Anal. Math. Phys. 3 (2013), no. 1, 1-19, DOI 10.1007/s13324-012-0035-4. MR.3015627

[Ebe13] Peter Ebenfelt, Partial rigidity of degenerate CR embeddings into spheres, Adv. Math. 239 (2013), 72-96, DOI 10.1016/j.aim.2013.02.011. MR.3045142

[EHZ05] Peter Ebenfelt, Xiaojun Huang, and Dmitri Zaitsev, The equivalence problem and rigidity for hypersurfaces embedded into hyperquadrics, Amer. J. Math. 127 (2005), no. 1, 169191. MR2115664 (2006c:32047)

[GLV11] Dusty Grundmeier, Jiří Lebl, and Liz Vivas. Bounding the rank of hermitian forms and rigidity for CR mappings of hyperquadrics. arxiv:1110.4082v2[mathCV], 2011.

[Gre89] Mark Green, Restrictions of linear series to hyperplanes, and some results of Macaulay and Gotzmann, Algebraic curves and projective geometry (Trento, 1988), Lecture Notes in Math., vol. 1389, Springer, Berlin, 1989, pp. 76-86, DOI 10.1007/BFb0085925. MR:1023391(90k:13021) 
[Gre10] Mark L. Green, Generic initial ideals, Six lectures on commutative algebra (Bellaterra, 1996), Progr. Math., vol. 166, Birkhäuser, Basel, 1998, pp. 119-186. MR1648665 (99m:13040)

[Gru11] Dusty Grundmeier, Signature pairs for group-invariant Hermitian polynomials, Internat. J. Math. 22 (2011), no. 3, 311-343, DOI 10.1142/S0129167X11006775. MR.2782691 (2012c:32031)

[HJY13] Xiaojun Huang, Shanyu Ji, and Wanke Yin, On the third gap for proper holomorphic maps between balls. Mathematische Annalen, pages 1-28, 2013.

[HL13] Jennifer Halfpap and Jiří Lebl. Signature pairs of positive polynomials. Bull. Inst. Math. Acad. Sin. (N.S.), 8(2):169-192, 2013.

[Hua99] Xiaojun Huang, On a linearity problem for proper holomorphic maps between balls in complex spaces of different dimensions, J. Differential Geom. 51 (1999), no. 1, 13-33. MR 1703603 (2000e:32020)

[Mac27] F. S. Macaulay, Some properties of enumeration in the theory of modular systems. Proc. Lond. Math. Soc., 26:531-555, 1927.

[Qui68] Daniel G. Quillen, On the representation of hermitian forms as sums of squares, Invent. Math. 5 (1968), 237-242. MR0233770 (38 \#2091)

[TY06] Wing-Keung To and Sai-Kee Yeung, Effective isometric embeddings for certain Hermitian holomorphic line bundles, J. London Math. Soc. (2) 73 (2006), no. 3, 607-624, DOI 10.1112/S0024610706022708. MR2241969 (2008e:32027)

Department of Mathematics, University of Michigan, Ann Arbor, Michigan 48109

E-mail address: grundmer@umich.edu

Current address: Department of Mathematical Sciences, Ball State University, Muncie, Indiana 47306

E-mail address: deg@bsu.edu

Department of Mathematical Sciences, University of Montana, Missoula, Montana 59812

E-mail address: halfpap@mso.umt.edu 